\title{
Substance-Induced Sleep Disorder
}

National Cancer Institute

\section{Source}

National Cancer Institute. Substance-Induced Sleep Disorder. NCI Thesaurus. Code C95079.

A sleep disorder (insomnia, hypersomnia, or parasomnia) for which there is strong evidence that the disorder is etiologically linked to the direct physiological effects of a substance (i.e., a drug of abuse, a medication, or toxin exposure). 